\title{
Rapid on-site evaluation of transbronchial aspirates: randomised comparison of
}

\section{two methods}

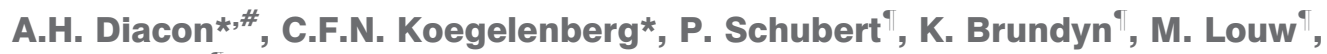 \\ C.A. Wright ${ }^{\top}$ and C.T. Bolliger*
}

ABSTRACT: The value of different staining methods for rapid analysis of transbronchial needle aspirates during bronchoscopy has not been explored.

In the present study, we compared a Papanicolaou-based rapid stain, prepared by a technologist and read by a cytopathologist, and a Wright-Giemsa-based rapid stain, prepared and read by a cytopathologist alone. Gold standard was the final laboratory report issued on each aspirate.

We harvested 827 aspirates from 218 target sites in 126 consecutive patients. At least one positive aspirate was found in 99 (79\%) patients. In those 99 patients, 288 of 574 (50\%) aspirates were positive for neoplastic $(83 \%)$ or non-neoplastic $(17 \%)$ disease. False-negative aspirates and target sites were more frequent with the rapid Wright-Giemsa than with the rapid Papanicolaou stain (14.2 versus $7.3 \%, p=0.008$, and 13.7 versus $3.6 \%, p=0.021$, respectively). The sensitivity of the Wright-Giemsa-based and Papanicolaou-based rapid stains for detecting diagnostic material was 93 and $100 \%$ in patients, 83.1 and $95.5 \%$ in target sites, and 72.8 and $84.9 \%$ in aspirates, respectively. Specificity was $100 \%$ for both methods in patients and target sites, and 90.4 and $95 \%$ in aspirates.

We concluded that a Papanicolaou-based stain has superior yield and accuracy to a WrightGiemsa-based stain for rapid on-site evaluation of transbronchial needle aspirates.

\section{KEYWORDS: Biopsy, bronchoscopy, cytodiagnosis, fine-needle, lung neoplasms}

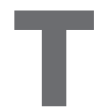
ransbronchial needle aspiration (TBNA) via flexible bronchoscopy (FB) is a wellestablished sampling method for a variety of neoplastic and non-neoplastic lung lesions [1]. Its ability to establish a diagnosis and to permit staging in a single intervention has made TBNA the key technique for the evaluation of patients with suspected lung cancer [2-4]. Endobronchial ultrasound and rapid on-site evaluation (ROSE) have been shown to improve TBNA yield [5-9]. ROSE-TBNA provides the bronchoscopist with real-time guidance and allows for termination of sampling when the diagnostic objective has been met. This increases patient comfort and reduces cost $[8,10]$. A formal off-site review involving routine and ancillary staining methods, e.g. for microorganisms and immunocytochemistry is obligatory to confirm provisional ROSE results.

Experienced bronchoscopists harvest TBNA samples in quick succession by repetitively aspirating multiple target sites to minimise false-negative results. To fulfil its purpose, a ROSE procedure must keep up with the pace of sampling. Onsite staining methods must therefore find a compromise between speed, ease of preparation and staining quality. Commonly used fast stains are modified versions of standard laboratorybased staining methods. Wright-Giemsa-based rapid stains are water-based and provide mainly cytoplasmic definition on air-dried slides. Such procedures are not labour-intensive and allow staining and evaluation by a single person. Alcohol-based modified Papanicolaou stains on alcohol-fixed slides are more time consuming but provide superior demonstration of nuclear features. This comes at the cost of requiring on-site hands-on assistance for the cytopathologist.

The practical utility of different ROSE stains is not well standardised and validated. This study investigated whether either of these two popular methods is superior in yield and accuracy of rapid on-site staining of transbronchial aspirates.

\section{METHODS}

Patients, interventions and diagnoses

Consecutive patients scheduled for FB with TBNA were randomised to ROSE with either one of the rapid-staining methods. Three pulmonologists
AFFILIATIONS

Depts of *Medicine,

\#Biomedical Sciences, and

-Anatomical Pathology and National

Health Laboratory Service, Tygerberg

Academic Hospital, University of

Stellenbosch, Cape Town, South Africa.

CORRESPONDENCE

A.H. Diacon

Division of Physiology

Dept of Biomedical Sciences

PO Box 19063

7505 Tygerberg

South Africa

E-mail: ahd@sun.ac.za

Received:

March 282009

Accepted after revision:

Nov 092009

First published online:

Nov 192009 
experienced in TBNA performed or supervised all procedures using standard video bronchoscopes (Exera; Olympus, Hamburg, Germany) and standard TBNA for cytological specimens (Bard, Billerica, MA, USA) under topical anaesthesia (lidocaine 1\%) and conscious sedation (midazolam intravenously as needed). Target sites were arbitrarily subdivided into tracheobronchial sites (regions 1 to 4 according to the American Thoracic Society (ATS) system [11]), bronchial sites (all other sites in visible range) and peripheral sites. It was recommended that operators harvest at least three successive aspirates per target site in close proximity. TBNA sampling ended when all target sites had been aspirated or upon ROSE confirmation of two diagnostic aspirates. All patients gave written informed consent.

\section{Staining methods}

One of two cytopathologists (M. Louw and K. Brundyn) performed ROSE. Each aspirate was immediately expressed onto numbered glass slides, and smears were made and stained with either a rapid modified Wright-Giemsa stain (G-ROSE) or a modified rapid Papanicolaou stain (P-ROSE). G-ROSE (Rapidiff; Clinical Sciences Diagnostic CC, Johannesburg, South Africa) on unfixed air-dried slides involved three steps and took $45 \mathrm{~s}$ to complete. Staining and on-site evaluation was performed by a cytopathologist on wet stains without assistance. For P-ROSE, smears were spray-fixed (ether/alcohol with poly-ethylene-glycol; Fencott, Sangene Products, Cape Town, South Africa) and stained on-site with a procedure involving 15 steps in $3 \mathrm{~min}$. Slides were stained and coverslipped by a technologist on-site and handed over to the cytopathologist for evaluation. Each slide was reported separately in theatre for: 1) the presence of diagnostic material; 2 ) the presence of neoplastic versus non-neoplastic disease; and 3) diagnostic subclassification of neoplastic lesions into lymphoma, epithelial malignancies (small cell or nonsmall cell lung cancer) or unspecified malignant cells.

\section{Statistical aspects}

An independent person provided sequentially numbered closed envelopes to randomise procedures into equal groups in blocks of six, which were opened at the Pathology Dept of our institute (National Health Laboratory Service, Cape Town, South Africa) immediately before each bronchoscopy. For each procedure, a separate trolley equipped with the staining tools for either method was used. Each ROSE slide was reviewed off-site by a different cytopathologist (M. Louw or K. Brundyn). A third, senior cytopathologist (C.A. Wright) was available to resolve diagnostic discrepancies. Formal proficiency training was not considered necessary as both stains were routinely employed before the study. To detect a potential training bias, we compared the number of falsenegative ROSE results with either stain in the first and second half of the study. A positive TBNA was defined as an aspirate that contained diagnostic material on the final laboratory report for each aspirate. A positive target site and positive FB were defined as a site or FB with at least one positive TBNA.

The primary outcome was the accuracy of each ROSE method to predict a positive TBNA. The secondary outcomes were prediction of a positive target site, positive FB and the accuracy of the tissue diagnosis. Sample size was estimated by extrapolating from recent experience at our institution (Tygerberg Hospital, Cape Town, South Africa) [8]. We conservatively assumed a proportion of positive passes $(50 \%)$, an average number of TBNA performed per patient $(n=7)$, and a proportion of patients with at least one positive TBNA $(80 \%)$. This would allow detection of an increase in false-negative aspirates from 5 to $15 \%$ with 63 patients per group (significance level $\mathrm{p}<0.05$, power 0.80 ). Proportional data were analysed using Pearson's Chi-squared test or Fisher's exact test in case of very small counts $(\leqslant 5)$. A p-value of $<0.05$ was considered significant. Two-sided tests were used. Values are presented as mean $\pm \mathrm{SD}$, unless stated otherwise.

\section{RESULTS}

\section{Patients, diagnoses and interventions}

The study included 126 consecutive patients who underwent FB with TBNA (age 55.1 \pm 14.7 yrs, 61\% male). From 218 target sites (121 tracheobronchial (56\%), 88 other sites in visible range $(40 \%)$, and nine peripheral sites $(4 \%))$, we sampled 827 needle passes $(6.6 \pm 2.8$ per patient; $3.8 \pm 1.6$ per target site). The final bronchoscopic diagnosis was neoplastic disease in $85(68 \%)$ patients and non-neoplastic disease in 17 (12\%) patients. In 24 (19\%) patients, FB did not yield a diagnosis (table 1). For the comparative analysis of ROSE performance, we did not include $27 \mathrm{FB}$ results that had only negative TBNA and seven FB results that were only positive on microbiological culture of an aspirate. The distribution of procedural parameters between G-ROSE and P-ROSE is shown in table 2. No significant differences were found between groups. The distribution of GROSE and P-ROSE among bronchoscopists did not differ significantly (data not shown).

\section{Prediction of positive TBNA}

The accuracy of both ROSE procedures for predicting positive TBNA was high (table 3), with P-ROSE being slightly superior to G-ROSE at all levels. False-negative readings were

\begin{tabular}{lcc} 
TABLE 1 Patients and bronchoscopic diagnosis & \\
& Diagnosis & TBNA yield \\
\hline & & \\
All & $126(100)$ & $99(79)$ \\
Neoplastic disease & $85(68)$ & $82(96)$ \\
Non-small cell lung cancer & $75(60)$ & $72(96)$ \\
Adenocarcinoma & $36(29)$ & \\
Squamous cell carcinoma & $14(11)$ & $8(100)$ \\
Undifferentiated carcinoma & $25(20)$ & $2(100)$ \\
Small cell lung cancer & $8(6)$ & $17(100)$ \\
Lymphoma & $2(2)$ & \\
Non-neoplastic disease & $17(13)$ & \\
Tuberculosis & $14(11)$ & \\
Sarcoidosis & $3(2)$ & \\
Undiagnosed & $24(19)$ &
\end{tabular}

Data are presented as $\mathrm{n}(\%)$. \#: at least one diagnostic aspirate; $\because$ granulomatous necrosis on transbronchial needle aspiration (TBNA) and positive culture for Mycobacterium tuberculosis $(n=5)$, positive culture for M. tuberculosis only $(n=7)$, and granulomatous necrosis only $(n=2)$. 


\begin{tabular}{cccc}
\hline TABLE 2 & $\begin{array}{l}\text { Transbronchial needle aspiration sites and } \\
\text { needle passes }\end{array}$ \\
& Both methods & G-ROSE & P-ROSE \\
\hline All procedures & $126(100)$ & $63(50)$ & $63(50)$ \\
Sites aspirated & $218(100)$ & $108(50)$ & $110(50)$ \\
Lymph node & $121(56)$ & $58(54)$ & $63(57)$ \\
Other & $97(44)$ & $50(46)$ & $47(43)$ \\
Needle passes & $827(100)$ & $401(48)$ & $426(52)$ \\
Lymph node & $483(58)$ & $229(57)$ & $254(60)$ \\
Other & $344(42)$ & $172(43)$ & $172(40)$ \\
Diagnostic procedures & $92(73)$ & $43(47)$ & $49(53)$ \\
Neoplastic & $82(89)$ & $38(88)$ & $44(90)$ \\
Non-neoplastic & $10(11)$ & $5(12)$ & $5(10)$ \\
Sites aspirated & $157(100)$ & $73(46)$ & $84(54)$ \\
Lymph node & $109(69)$ & $39(53)$ & $46(55)$ \\
Other & $48(31)$ & $34(47)$ & $38(45)$ \\
Needle passes & $574(100)$ & $261(45)$ & $313(55)$ \\
Lymph node & $326(57)$ & $146(56)$ & $180(58)$ \\
Other & $248(43)$ & $115(44)$ & $133(42)$ \\
\hline
\end{tabular}

Data are presented as n (\%). G-ROSE: Wright-Giemsa-based rapid on-site evaluation; P-ROSE: Papanicolaou-based rapid on-site evaluation.

significantly more frequent with G-ROSE at target site level (13.7 versus $3.6 \% ; \mathrm{p}=0.021)$ and at the level of single aspirates $(14.2$ versus $7.3 \%$; $\mathrm{p}=0.008)$. Overall sensitivities for $\mathrm{G}$-ROSE and PROSE were: 72.8 and $84.9 \%$ at single pass, 83.1 and $95.5 \%$ at target site, and 93.0 and $100 \%$ at bronchoscopy level. Falsepositive on-site readings were rare $(<1 \%)$ with both methods and occurred only at single aspiration level. There was no significant difference in false-negative readings between the first and second half of the study (data not shown).

\section{Prediction of a specific diagnosis}

Overall, TBNA was diagnostic in 99 out of 126 (79\%) procedures, and in 82 out of 85 (96\%) patients with neoplastic disease. G-ROSE failed to recognise a diagnostic sample and hence no diagnosis was provided in three out of $43(7 \%)$ patients with positive TBNA (nonsmall cell lung cancer (NSCLC) $n=1$, small cell lung cancer (SCLC) $n=1$, granulomatous inflammation with positive culture for Mycobacterium tuberculosis $\mathrm{n}=1$ ). P-ROSE provided a provisional diagnosis for all 49 patients with positive TBNA. Both ROSE procedures accurately discriminated between neoplastic and non-neoplastic disease. A subclassification into lymphoma, SCLC and NSCLC was made in 35 out of 43 (81\%) FB with G-ROSE, and in 41 out of $49(82 \%)$ FB with P-ROSE. All diagnoses made were accurate. G-ROSE was not diagnostic in three negative FB and five FB with unspecified neoplastic cells (all NSCLC). Eight cases were classified as unspecified neoplastic cells with P-ROSE (NSCLC $n=5$, SCLC $n=3$ ). None of these differences was statistically significant.

\section{DISCUSSION}

The present randomised comparison of two on-site staining methods for TBNA in patients representative for clinical

\begin{tabular}{|c|c|c|c|c|}
\hline \multirow[t]{2}{*}{ TABLE 3} & \multicolumn{4}{|c|}{$\begin{array}{l}\text { Accuracy of rapid on-site evaluation (ROSE) in } \\
\text { procedures with at least one positive } \\
\text { transbronchial needle aspiration (TBNA) }\end{array}$} \\
\hline & All & G-ROSE & P-ROSE & $p$-value \\
\hline Total procedures & 126 & 63 & 63 & \\
\hline $\begin{array}{l}\text { Procedures with positive } \\
\text { TBNA }\end{array}$ & $92(100)$ & 43 & 49 & 0.228 \\
\hline True positive & 89 (96.7) & $40(93.0)$ & $49(100)$ & 0.078 \\
\hline False positive & $0(0)$ & $0(0)$ & $0(0)$ & $\mathrm{N} / \mathrm{A}$ \\
\hline True negative & $0(0)$ & $0(0)$ & $0(0)$ & N/A \\
\hline False negative & $3(3.3)$ & $3(7.0)$ & $0(0)$ & 0.122 \\
\hline Sensitivity \% & 96.7 & 93.0 & 100 & \\
\hline Specificity \% & 100 & 100 & 100 & \\
\hline Sites aspirated ${ }^{\#}$ & 157 & 73 & 84 & \\
\hline Off-site positive & $126(80.3)$ & 59 (80.8) & 67 (79.8) & 0.868 \\
\hline True positive & 113 & 49 & 64 & 0.207 \\
\hline False positive & 0 & 0 & 0 & $\mathrm{~N} / \mathrm{A}$ \\
\hline True negative & 31 & 14 & 17 & 0.868 \\
\hline False negative & 13 & 10 & 3 & 0.017 \\
\hline Sensitivity \% & 89.7 & 83.1 & 95.5 & \\
\hline Specificity \% & 100 & 100 & 100 & \\
\hline Needle passes ${ }^{\#}$ & 574 & 261 & 313 & \\
\hline Off-site positive & $288(50.2)$ & $136(52.1)$ & $152(48.6)$ & 0.398 \\
\hline True positive & 228 & 99 & 129 & 0.423 \\
\hline False positive & 20 & 12 & 8 & 0.184 \\
\hline True negative & 266 & 113 & 153 & 0.181 \\
\hline False negative & 60 & 37 & 23 & 0.008 \\
\hline Sensitivity \% & 79.2 & 72.8 & 84.9 & \\
\hline Specificity \% & 93.0 & 90.4 & 95.0 & \\
\hline
\end{tabular}

Data are presented as $n(\%)$ or $n$, unless otherwise stated. G-ROSE: WrightGiemsa-based ROSE; P-ROSE: Papanicolaou-based ROSE; N/A: not applicable. ${ }^{\#}$ : in procedures with at least one positive TBNA. The $p$-values provide a comparison between G-ROSE and P-ROSE.

practice confirmed the high sensitivity of ROSE, with both methods combined predicting $96.7 \%$ of positive procedures, $89.7 \%$ of positive target sites and $79.2 \%$ of positive aspirates. Compared to the Wright-Giemsa-based rapid stain, the Papanicolaou-based rapid stain provided better diagnostic accuracy. Owing to significantly fewer false-negative aspirates, P-ROSE predicted $12.4 \%$ more positive target sites and $7 \%$ more positive procedures than G-ROSE, at the cost of an additional on-site cytology technologist. False-positive aspirates were rare with both methods. Both stains discriminated correctly between neoplastic and non-neoplastic disease, and both stains accurately differentiated between lymphoma, SCLC and NSCLC in $>80 \%$ of cases. These findings contribute to the standardisation of ROSE for TBNA.

To our knowledge, this is the first randomised comparison of ROSE staining methods for TBNA. The $84 \%$ on-site yield with P-ROSE was consistent with a previous study from our group, where P-ROSE recognised $85 \%$ of positive TBNA [8]. Other studies have associated ROSE with a higher yield of TBNA $[6,7]$, better aspirate quality $[6,12]$, educational value [12], improved quality of service $[7,8,10]$ and cost-effectiveness, 

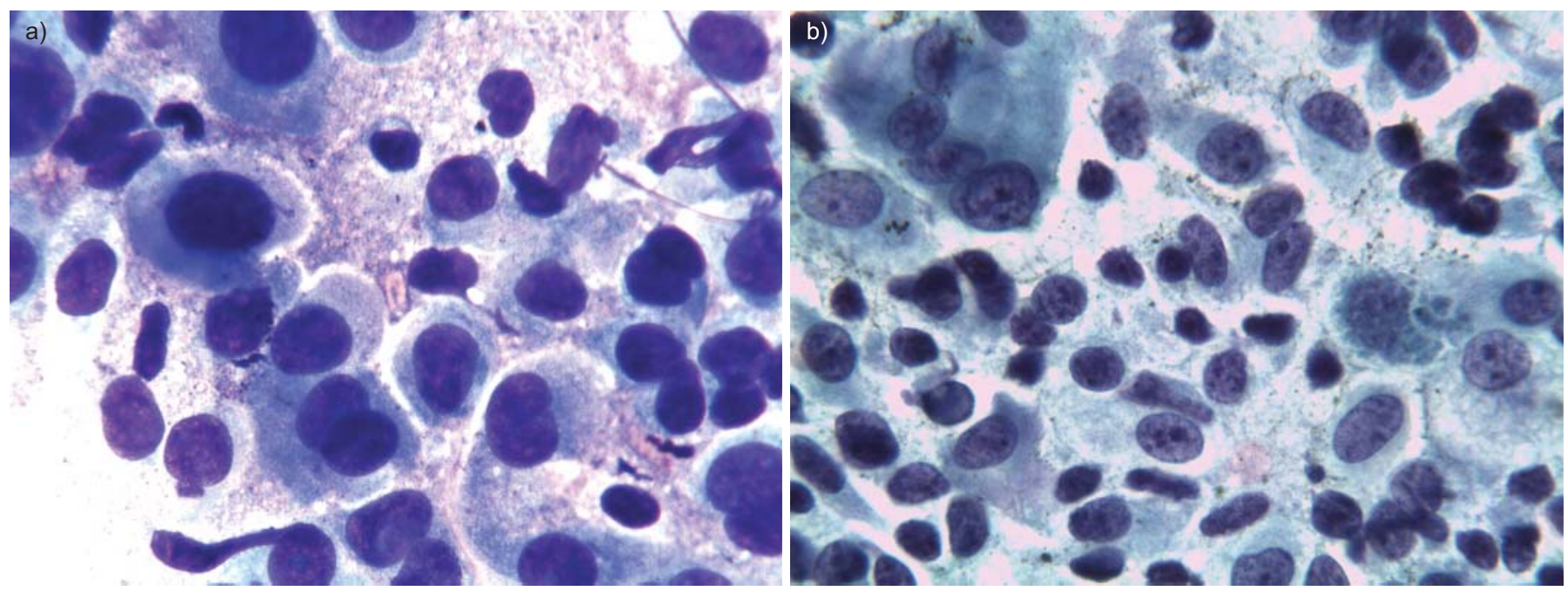

FIGURE 1. Transbronchial needle aspirate with adenocarcinoma, stained with different methods on-site and photographed on-site. a) The Wright-Giemsa-based rapid stain demonstrates cytoplasm more clearly and shows dark pleomorphic nuclei, but chromatin quality is poor. b) The Papanicolaou-based rapid stain demonstrates crisp nuclear morphology with irregular nuclear membranes and nucleoli, and is hence better suited to diagnosis of malignancy. Magnification $\times 1,000$.

due to shorter procedure time and fewer samples submitted for analysis $[8,10]$. In our experience, the bronchoscopist in routine clinical practice benefits from ROSE in two ways. First, ROSE can determine when sufficient diagnostic material has been harvested. This is most helpful if stepwise aspiration of nodal stations from the highest-rated potentially involved region down to the primary tumour is required for staging of suspected bronchogenic carcinoma. Demonstration of positive N2 or N3 lymph nodes with TBNA avoids further, unnecessary exploration, with all the associated morbidity and cost [2, $8,10,11]$. Secondly, ROSE assists in selecting appropriate additional sampling modalities during FB. The wide differential diagnosis of enlarged mediastinal lymph nodes can be considerably narrowed by ROSE, which will provide real-time guidance as to whether sampling can be terminated (when bronchogenic carcinoma cells are harvested) or should continue for confirmation of lymphoma (additional TBNA for flow cytometry, large bore TBNA for histology), sarcoidosis-type granulomatous inflammation (transbronchial and mucosal biopsies, bronchoalveolar lavage) or granulomatous inflammation with necrosis as seen in tuberculosis (specimens for mycobacterial culture).

Off-site confirmation of all ROSE diagnoses is mandatory to avoid errors in diagnosis and clinical handling of patients. Interestingly, the few false-positive aspirates in the present study did not cause a target site or a procedure to be false positive. The study followed the rule that a procedure was terminated when at least two concordant positive on-site readings were harvested. This ensured that each false-positive aspirate was backed up with a true-positive aspirate analysed with the same method, which suggests that false-positive readings most likely occur by overcalling highly suspicious material on-site. It could be seen as a weakness of the present study that FB were randomised and not single aspirates, but in practice it was not feasible to mobilise two different on-site staining teams for each FB. False-negative readings can occur due to a combination of limited examination time and paucity of diagnostic material on an aspirate but may also be due to suboptimal stain quality. The majority of diagnoses made in bronchoscopy practice are bronchogenic carcinomas, and consequently the accuracy of ROSE will mostly depend on the ability of the stain to demonstrate changes associated with malignancy. In well-differentiated tumours such as bronchiolar alveolar carcinoma these may be subtle nuclear abnormalities, highly dependent on optimal preparation and staining of the slides. While G-ROSE is faster, it is also known for its ability to demonstrate cytoplasmic features (fig. 1a), whereas P-ROSE is more complex but is superior in demonstrating the nuclear features associated with malignancy (fig. 1b). If all other factors are standardised as they were in the present study, it is reasonable to speculate that this property makes $\mathrm{P}$ ROSE the superior stain for ROSE-TBNA, albeit at the price of technical assistance for its preparation.

Does the on-site stain really matter in clinical practice? The answer will depend on local factors such as cost, availability of trained cytology staff and geographical proximity of services. The scientifically minded pathologist will insist that there is a measurable benefit of P-ROSE, that the best tools must be used in the laboratory, and will drive for an additional person to be provided for on-site assistance. The cost-conscious hospital manager will argue that using P-ROSE instead of G-ROSE will only detect one more positive target site every eight patients at best. The clinician, most aware that thorns and roses grow on the same tree, should declare that ROSE is an essential service and that it is best delivered with a Papanicolau-based fast stain.

\section{SUPPORT STATEMENT}

A.H. Diacon was supported by the Respiratory Research Unit of the University of Stellenbosch (Cape Town, South Africa).

\section{STATEMENT OF INTEREST}

None declared. 


\section{ACKNOWLEDGEMENTS}

We thank the bronchoscopy staff and the cytology technologists of the Tygerberg Hospital and the National Health Laboratory Service (Cape Town, South Africa) for their assistance.

\section{REFERENCES}

1 Mazzone P, Jain P, Arroliga AC, et al. Bronchoscopy and needle biopsy techniques for diagnosis and staging of lung cancer. Clin Chest Med 2002; 23: 137-158.

2 Minai OA, Dasgupta A, Mehta AC. Transbronchial needle aspiration of central and peripheral lesions. In: Bolliger CT, Mathur PN, eds. Interventional bronchoscopy. Basel, Karger, 2000; pp. 66-79.

3 Gasparini S, Silvestri GA. Usefulness of transbronchial needle aspiration in evaluating patients with lung cancer. Thorax 2005; 60: 890-891.

4 Medford AR, Bennett JA, Free CM, et al. Mediastinal staging procedures in lung cancer: EBUS, TBNA and mediastinoscopy. Curr Opin Pulm Med 2009; 15: 334-342.
5 Herth FJ, Becker HD, Ernst A. Ultrasound-guided transbronchial needle aspiration: an experience in 242 patients. Chest 2003; 123: 604-607.

6 Davenport RD. Rapid on-site evaluation of transbronchial aspirates. Chest 1990; 98: 59-61.

7 Diette GB, White P, Terry P, et al. Utility of on-site cytopathology assessment for bronchoscopic evaluation of lung masses and adenopathy. Chest 2000; 117: 1186-1190.

8 Diacon AH, Schuurmans MM, Theron J, et al. Utility of rapid onsite evaluation of transbronchial needle aspirates. Respiration 2005; 72: $182-188$.

9 Herth FJ, Eberhardt R, Ernst A. The future of bronchoscopy in diagnosing, staging and treatment of lung cancer. Respiration 2006; 73: 399-409.

10 Baram D, Garcia RB, Richman PS. Impact of rapid on-site cytologic evaluation during transbronchial needle aspiration. Chest 2005; 128: 869-875.

11 Mountain CF, Dresler CM. Regional lymph node classification for lung cancer staging. Chest 1997; 111: 1718-1723.

12 Hsu LH, Liu CC, Ko JS. Education and experience improve the performance of transbronchial needle aspiration: a learning curve at a cancer center. Chest 2004; 125: 532-540. 